\title{
Instrumentos de medición del desarrollo en Chile
}

\author{
PAULA BEDREGAL ${ }^{1}$ \\ 1. Departamento de Salud Pública, Pontificia Universidad Católica de Chile.
}

Palabras clave: Desarrollo, instrumentos, indicadores, salud, escolar.

El crecimiento y desarrollo humano es un proceso continuo a lo largo de la vida, influido por las experiencias de la persona y donde cada etapa se asienta en la anterior. Por lo tanto, este proceso ocurre en la interacción del niño, como organismo biológico, con el medio inmediato y la interacción entre el sistema social y el ambiente del niño ${ }^{1}$.

En el desarrollo humano integral es posible analizar dos tipos de cambios: cuantitativo y cualitativo. El primero corresponde a los cambios sucedidos en cantidad en el niño, y que implican el crecimiento físico; el segundo corresponde a la aparición de nuevos fenómenos que se suelen expresar a través de la conducta. En el desarrollo cualitativo se suele diferenciar al menos 5 componentes: motor, cognitivo, del lenguaje, emocional y social.

Gran parte de la investigación en el área de desarrollo se ha centrado en su descripción, para lo cual se han desarrollado múltiples instrumentos de medición, los que se sustentan en la elaboración de estándares para los diversos componentes. Del mismo modo se han creado indicadores que permiten dar cuenta de manera parcial de la situación de niños y niñas.

Desde el punto de vista de la medición es interesante destacan que la mayor parte de la información disponible internacionalmente corresponde a la situación del desarrollo físico de los niños. Existe escasa información sistematizada sobre los otros 5 componentes, en particulares en los menores de 4 años. Esto por que el ingreso a la educación formal contempla en muchos países, sistemas de monitoreo de la calidad, que involucran explorar al menos el desarrollo cognitivo de los niños ${ }^{2}$.

Otro punto relevante cuando se habla de medición en desarrollo psicosocial, es comprender que los instrumentos utilizados no son realmente transculturales. Esto, por que la mayor parte de ellos, han sido diseñados, pensando en las habilidades que la sociedad occidental promueve. Numerosas investigaciones empíricas han destacado que una brecha en el desarrollo infantil no implica necesariamente desiguales niveles de logro con relación a una escala "universalmente" válida. En una importante proporción, los casos de desarrollo insuficiente serán el reflejo de la discordancia entre los patrones que definen el crecimiento y desarrollo en los grupos de pertenencia y aquellos establecidos como normas por el conjunto de la socie$\mathrm{dad}^{3}$. Por lo tanto, en algunos casos los indicadores pueden no tener significado para los grupos de pertenencia, ya que miden áreas poco relevantes para estos grupos, relegando otras importantes; sin embargo, el niño que se encuentra en esta situación, sin presentar un problema realmente, ve su vida afectada por que suele ser discriminado por "el quiebre simbólico entre su mundo de origen y el mundo exterior" 4 .

Correspondencia a:

Paula Bedregal

E-mail:pbedrega@med.puc.cl 
MEDICIÓN DEL DESARROLLO

Prácticamente todos los instrumentos de medición tienen como fundamento una teoría respecto de este proceso, lo que hace difícil comparar resultados entre estudios y contextos, de modo que cualquier intento de comparar resultados debería hacerse bajo los mismos supuestos teóricos de la medición.

Con estos antecedentes a continuación presentamos los instrumentos que han sido utilizados o se utilizan en Chile tanto por el sector salud como educación, excluyendo los instrumentos que se han utilizado en investigaciones empíricas.

\section{Los instrumentos e indicadores utilizados por el sector Salud chileno}

La mayor parte de los instrumentos e indicadores utilizados por el sector salud, provienen de las normas vigentes desarrolladas por el Ministerio de Salud, habitualmente en consulta a expertos.

Los indicadores de crecimiento físico se basan en estándares internacionales y consideran el método tradicional de medición del Peso, la Talla y la Circunferencia Craneana (hasta los dos años). En Chile, desde 1994 se utiliza para los menores de 6 años como patrón de referencia las curvas del NCHS, aplicadas en desviaciones estándares respecto de la media (y no en percentiles), las que sustituyeron al patrón SEMPE5. El diagnóstico nutricional poblacional se basa en el "diagnóstico nutricional integrado", utilizando los tres indicadores tradicionales (Peso/Edad, Talla/Edad y Peso/Talla), más los datos anamnésticos y la curva de crecimiento ${ }^{6}$. En el caso de los escolares, la evaluación queda a cargo de la JUNAEB, institución que como parte del Programa de Salud Escolar, realiza evaluaciones periódicas en cohortes sucesivas tanto del estado nutricional como detección de enfermedades crónicas, de niños asistentes a colegios municipales.

En cuanto a desarrollo psicosocial, hace aproximadamente dos décadas se incorporó en la atención de control de salud de niños menores de dos años, su evaluación mediante un instrumento de tamizaje validado en Chile (EEDP). Durante las primeras dos décadas, el problema de este instrumento fue su mala utilización y su poco uso. Esto en parte estuvo dado por la dificultad de los equipos de salud de contar con tiempo programado para esta activi$\mathrm{dad}^{7}$. Durante la década de los '90 se crearon las normas y se inició la implementación de un Programa de estimulación y evaluación del desarrollo psicomotor en niños menores de seis años, que utiliza como base a instrumentos estandarizados a nivel nacional y el uso de una escala de tamizaje clínico: la Pauta Breve de Evaluación del Desarrollo y EEDP ${ }^{8}$ para niños menores de 2 años y el TEPSI para niños entre 2 y 5 años $^{9}$ (tabla 1 ).

En el sector salud, destaca la aplicación de instrumentos para evaluar desarrollo realizado por el Programa de Desarrollo Psicomotor y de Estimulación Temprana realizado en Temuco, con apoyo de la Universidad Católica de Temuco y la Universidad de la Frontera. Este grupo ha utilizado para sus evaluaciones el EEDP y TEPSI. Su aplicación ha sido bajo un modelo de

Tabla 1. Instrumentos de desarrollo psicosocial usados en el sector salud

\begin{tabular}{|c|c|c|c|c|}
\hline Instrumento & Autores & Uso del test & Componentes evalúa & Calidad \\
\hline $\begin{array}{l}\text { EEDP (Escala para la } \\
\text { evaluación del desarrollo } \\
\text { psicomotor para niños } \\
\text { entre } 0 \text { y } 2 \text { años) }\end{array}$ & $\begin{array}{l}\text { Rodríguez, } \\
\text { Arancibia, } \\
\text { Undurraga }\end{array}$ & $\begin{array}{l}\text { Tamizaje. } \\
\text { Observación directa } \\
\text { (0 a } 2 \text { años) }\end{array}$ & $\begin{array}{l}\text { Motora: gruesa y fina, } \\
\text { coordinación, lenguaje y } \\
\text { social }\end{array}$ & $\begin{array}{l}\text { Baja sensibilidad y especifici- } \\
\text { dad en }<4 \text { meses. } \\
\text { Valor Predictivo Positivo }>4 \\
\text { meses } 97 \text { a } 100 \% \text {. } \\
\text { Muestra: no considera } \\
\text { distinción urbano, rural }\end{array}$ \\
\hline $\begin{array}{l}\text { TEPSI (Test de desarrollo } \\
\text { psicomotor de } 2 \text { a } 5 \text { años) }\end{array}$ & $\begin{array}{l}\text { Heussler, } \\
\text { Marchant }\end{array}$ & $\begin{array}{l}\text { Tamizaje. } \\
\text { Observación directa } \\
\text { niños de } 2 \text { a } 5 \text { años }\end{array}$ & $\begin{array}{l}\text { Motricidad, coordinación y } \\
\text { lenguaje }\end{array}$ & $\begin{array}{l}\text { Hay un } 29 \% \text { ítems considera- } \\
\text { dos fáciles; } 52 \% \text { medianos y } \\
19 \% \text { difíciles. } \\
\text { Muestra: no contempla } \\
\text { urbano/rural }\end{array}$ \\
\hline
\end{tabular}


intervención Clínico Pedagógico (incorporación de educadora de párvulos en el centro de salud primaria), el que ha sufrido modificaciones y ajustes por 10 años ${ }^{10}$. En la práctica clínica, para el seguimiento de niños prematuros y con déficit, se ha usado el test Griffith ${ }^{11}$ y el Bayley ${ }^{12}$.

Finalmente, con la implementación del programa Chile Crece Contigo en 2007, se ha estimulado en las guías técnicas, el uso del EEDP y TEPSI, con tiempo protegido para su aplicación en la cohorte de niños que ingresan al Programa ${ }^{13}$. Adicionalmente se ha incluido una pauta de evaluación de una función psicológica (apego) a través de la pauta de observación de Massie-Campell. Este instrumento requiere de capacitación adicional y supervisión permanente. Evalúa estilos de apego en la díada madre niño o niña durante los controles de salud a los 4 y 12 meses. Permite evaluar la calidad de la relación vincular entre un "cuidador" y su bebé en situaciones de stress. El instrumento arroja 3 patrones de apego (seguro; evitante y ambivalente). En su versión inglesa tiene una confiabilidad entre examinadores de 0,83 a 0,84 . No hay estudios sobre validez discriminante ${ }^{14}$.

\section{Los instrumentos e indicadores utilizados por el sector Educación}

En el sector educación no se utiliza de manera regular instrumentos de evaluación del desarrollo en menores de 6 años. Desde el trabajo de JUNAEB es posible obtener información del Programa de Salud escolar dirigido a niños desde primero básico (7 años). Este programa entrega información antropométrica. Adicionalmente, en el ámbito de salud mental se ha implementado la evaluación de la conducta mediante autoreporte paterno, utilizando el Pediatric Symptom Checklist, (M. Jellineck, Boston) y el TOCA-R que evalúa riesgos conductuales (Shepard Kellom, U. John Hopkins) ${ }^{15-18}$.

Durante 2005 se editaron por parte del MINEDUC los Estándares de Aprendizaje para la Educación Parvularia ${ }^{19}$. Estos, permiten identificar áreas de evaluación para niños entre 0 y 6 años, que requerirán de instrumentos apropiados para su evaluación. Estos estándares cuentan como marco de referencia "Las Bases
Curriculares para la Educación Parvularia" y como soporte metodológico el modelo de "Habilidades de Progresión". Los estándares están organizados en ejes y núcleos. El primero corresponde a los resultados esperados que se asocian a componentes del desarrollo específicos. El segundo aspecto se relaciona con los núcleos de aprendizaje identificándose: autonomía, identidad, convivencia, lenguaje verbal, lenguaje artístico, relaciones lógico-matemáticas y cuantificación; seres vivos y entorno, grupos humanos, sus formas de vida y acontecimientos relevantes; y disposición al aprendizaje. Estos estándares no incluyen el desarrollo físico.

Otros instrumentos disponibles y validados en Chile son: el Developmental Screening Test, estandarizado en Chile (DDST) ${ }^{20}$; el Inventario de problemas conductuales y emocionales $(\text { IPCS })^{21} ; \mathrm{y}$ el Test de vocabulario en imágenes $(\text { TEVI })^{23}$.

El DDST o DENVER es un test de tamizaje de aplicación individual para población entre 1 mes y 6 años, que registra la conducta del niño informada y observada. Entrega un perfil para las áreas de motricidad gruesa, fina, adaptativa, lenguaje y conducta personal-social. La validación fue sobre la base de 2321 niños de nivel socioeconómico bajo, medio y alto; no considera población urbano/rural.

El IPCS es un instrumento de tamizaje para evaluar a niños de 3 a 5 años cuyo objetivo es detectar problemas conductuales y socioemocionales, evaluar problemas maternos y situaciones estresantes. Se basa en el reporte de los padres. La validación se realizó con una muestra de 309 niños de la Región Metropolitana de tres niveles socioeconómicos, sin contemplar diferencias urbano/rurales.

Por último, el TEVI es un instrumento de tamizaje para evaluar el nivel de comprensión de vocabulario pasivo para niños entre 2 a 16 años. Se aplica a los niños directamente y requiere de un muy buen entrenamiento. Su validación fue en Concepción (Sur de Chile) sobre una muestra de 1040 niños de estratos medios $\mathrm{y}$ altos urbanos.

Otras experiencias que han utilizado diversos instrumentos de medición se adjuntan en la tabla $2^{20}$.

Finalmente, la Pontificia Universidad Católi- 
Tabla 2. Experiencias chilenas evaluadas

\begin{tabular}{lll}
\hline Iniciativa & Grupo evaluado & Instrumento utilizado \\
\hline $\begin{array}{l}\text { Centros comunitarios de atención } \\
\text { preescolar (CCAPS) } \\
\text { Padres e hijos }\end{array}$ & Niños de 2 a 5 años & INTEGRA (elaborado por la institución) \\
$\begin{array}{l}\text { Programa de Mejoramiento de la Infancia } \\
\text { (CASH-PMI) }\end{array}$ & Niños de 4 a 6 años 5 a 6 años & TEPSI + Pauta de comportamiento socioafectivo \\
$\begin{array}{l}\text { Evaluación de centros INTEGRA } \\
\text { Niños de } 3 \text { a } 4 \text { años }\end{array}$ & $\begin{array}{l}\text { Pauta observación actividades pedagógicas } \\
\text { (cognición y socioemocional) }\end{array}$ \\
& & $\begin{array}{l}\text { Prueba K-ABC (Kaufman \& Kaufman, 1983): } \\
\text { Intelligence Test de inteligencia de WPPSI } \\
\text { (Wechsler, 1967) Auto-reporte de bienestar socio- } \\
\text { emocional (Lira y cols, 2000) }\end{array}$ \\
$\begin{array}{l}\text { Nuevo currículum de INTEGRA para } \\
\text { primer ciclo }\end{array}$ & $\begin{array}{l}\text { Niños nivel sala cuna y } \\
\text { Inventario Battelle }\end{array}$ \\
\hline
\end{tabular}

ca con apoyo del BID propuso un instrumento de evaluación de desarrollo, con fines de tamizaje poblacional, para ser aplicado en población de 0 a 6 años. La construcción se basó en el análisis de los componentes deseados y aquellos ítems que mejor lo representaran. Se realizó durante el segundo semestre de 2005 la validación clínica en 165 niños, contrastando el instrumento con la evolución en desarrollo y los diagnósticos clínicos con base a la ficha clínica. Este ejercicio se realizó en un centro de salud privado de la Universidad, en que como parte del currículo se enfatizan aspectos de desarrollo infantil. Los resultados arrojaron una sensibilidad de $85,7 \%$ y una especificidad de $81 \%$. La confiabilidad interna por tramo de edad no fue muy favorable, debido a que cada área identifica una dimensión, que no necesariamente afecta a las demás. Esta fluctuó entre 0,45 (KD20) en el grupo de 3 a 6 meses y 0,82 grupo de 46 a 48 meses $^{22}$. Este instrumento se aplicó mediante la Encuesta Nacional de Calidad de Vida y Salud, durante el 2006, contando con resultados para el país y zonas urbanas rurales ${ }^{23}$.

\section{Conclusión}

En Chile, existen múltiples instrumentos de evaluación del desarrollo en uso, algunos de carácter genérico y otros específicos; algunos de carácter poblacional y otros clínicos. Cada uno de los instrumentos posee diferencias psicométricas importantes, y algunos no han sido totalmente validados en Chile y normados. Desde la perspectiva de política pública es necesario contar con una mirada de conjunto de estos instrumentos, aclarando su objetivo y sus implicancias para cada sector, como también para el desarrollo de políticas de infancia integradas.

\section{Referencias}

1.- Brofenbrenner $U$ : The Ecology of Human Development. Cambridge, MA Harvard University Press.

2.- OECD: Starting Strong II: Early Childhood Education and Care. OECD, 2006.

3.- McDermott R H: Varenne (1995) Culture as Disability. Anthropology \& Education Quarterly 26.

4.- Bedregal P, M. Pardo (2004) Desarrollo Infantil Temprano y Derechos del Niño. Serie reflexiones: Infancia y Adolescencia. Santiago-Chile: UNICEF.

5.- SEMPE: Surveillance de la croissance de l'enfant. References pratiques. Le Concours medical, 1984 Sup.: 43.

6.- Ministerio de Salud de Chile: Norma para el manejo ambulatorio de la malnutrición por déficit y exceso en el niño(a) menor de 6 años. MINSAL, 2005.

7.- Lira MI, Gálvez G: El programa de estimulación precoz diez años después de una experiencia piloto. En: M Cusminsky, E Moreno y N Suárez-Ojeda (Eds) Crecimiento y Desarrollo. OPS. Publicación Científica $\mathrm{N}^{\circ}$ 510, 383-404. 1988.

8.- Rodríguez S, Arancibia V, Undurraga C: Escala de evaluación de desarrollo psicomotor para niños entre 0 y 2 años. Santiago: Editorial Galdoc 1976.

9.- Heussler IM, Marchant T: Tepsi. Test de Desarrollo 
Psicomotor 2-5 años. Décima edición. Santiago: Ediciones Universidad Católica de Chile, 2003.

10.- Taller de Experiencias Innovativas para el Desarrollo de la Primera Infancia. CEPAL, marzo 2004.

11.- Griffiths R: The Abilities of Babies. University of London Press London, UK, 1967.

12.- Moore R, Fabres J, Bedregal P: Seguimiento de recién nacidos de muy bajo peso en un Centro Universitario en Chile. Ann Pediatr (España) 2003; 58 (supl 3): 21 .

13.- Ministerio de Salud de Chile: Manual para el Apoyo y Seguimiento del Desarrollo Psicosocial de niños y niñas. 2007.

14.- Bruce AB, Tagle R: Psychoeducational assessment of preschool children. NY: Routledge, 2007.

15.- George $M$, Siraquian $X$, Mores $R$ : Adaptación y validación de dos instrumentos de pesquisa de problemas de salud mental en escolares de $1^{\circ}$ básico. Revista de Psicología 1995; 5: 17-25.

16.- De la Barra F, Toledo V, Rodríguez J: Estudio de salud mental en dos cohortes de niños escolares de Santiago Occidente.I: prevalencia y seguimiento de problemas conductuales y cognitivos. Rev Chil NeuroPsiquiat 2002; 40: 9-21.

17.- De la Barra F, Toledo V, Rodríguez J: Estudio de salud mental en dos cohortes de niños escolares de Santiago Occidente. II: factores de riesgo familiares y escolares. Rev Chil Neuro-Psiquiat 2002; 40: 347-60.

18.- De la Barra F, Toledo V, Rodríguez J: Estudio de salud mental en dos cohortes de niños escolares de Santiago Occidente. III: predictores tempranos de problemas conductuales y cognitivos Rev Chil Neuropsiquiatr 2003; 4: 65-74.

19.- MINEDUC-CEDEP: Estándares de aprendizaje para la educación parvularia. Habilidades en progresión desde el nacimiento hasta los 6 años. Santiago, 2005.

20.- Himmel E: Estandarización y validación del Denver Developmental Screening Test (DDST). Pontificia Universidad Católica de Chile. Mimeo. 1994.

21.- Rodríguez S, Lira MI, Arancibia V, Bralic S: Inventario de problemas conductuales y socioemocionales: 3-5 años. Santiago: Ediciones Universidad Católica, 1996.

22.- Bedregal P, Scharager J, Breinbauer C, et al: El Desarrollo de un modelo para la evaluación de rezagos del desarrollo infantil de Chile. Rev Méd Chile 2007; 135: 3: 403-5.

23.- Ministerio de Salud de Chile: Departamento de Epidemiología. Subsecretaría de Salud Pública. Encuesta Nacional de Calidad de Vida y Salud 2006.

24.- Newborg J, Stock JR, Wnek L, et al: Battelle Developmental Inventory with recalibrated Technical Data and Norms: Screening Test Examiner's Manual. 2nd ed. Allen, TX: DLM, Inc; 1988/2006. 